\title{
Painful peripheral states and sympathetic blocks
}

\author{
L. L O H A N D P. W. NATHA N ${ }^{1}$ \\ From the National Hospital for Nervous Diseases, Queen Square, London
}

S U MMARY In various chronic painful states, the sympathetic nerve supply was blocked either by injecting the sympathetic chain and ganglia with local anaesthesia or by the injection of guanethidine during occlusion of the circulation. There was a striking relation between the presence of hyperpathia and the relief of pain by the blocks. The sympathetic block was unlikely to relieve the pain unless hyperpathia accompanied the pain; when hyperpathia was present, a sympathetic block relieved both the constant pain and the hyperpathia. The effectiveness of the guanethidine blocks shows that the pain and the hyperpathia are maintained by the emission of noradrenaline in the periphery. The facts related to the sympathetic system and sensibility are discussed.

In the first world war, French surgeons, under the guidance of René Leriche, introduced sympathectomy for causalgia. In spite of this demonstration of a relation between pain and the sympathetic supply to a region, the satisfactory treatment of causalgia by sympathectomy has not served to explain why sympathectomy cures the condition; and we still know no more about the mechanisms involved than we did at the end of that war. Since that time, the subject has been advanced a little by two contributions in the second world war, those of Doupe et al. (1944) and Nathan (1947). Nathan showed that causalgia is not due to vascular stasis, vasoconstriction, or vasodilatation, and that it is not due to centripetal conduction to the spinal cord in sympathetic fibres. Doupe et al. proposed the following hypothesis, which was supported by Nathan: an artificial synapse is formed at the site of the lesion on the peripheral nerve; inpulses leaving the spinal cord in sympathetic fibres are switched at the artificial synapse by ephaptic transmission to small somatic afferent fibres; pain occurs when impulses pass along sympathetic fibres and return along these small myelinated and unmyelinated afferent nerve fibres.

However, the introduction of Hannington-Kiff's method (1974) of blocking the sympathetic nerve endings has made it apparent that this previous hypothesis is wrong. If the artificial synapse hypo-

\footnotetext{
${ }^{1}$ Member of the external scientific staff, Medical Research Council.

Address for reprint requests: Dr P. W. Nathan, National Hospital for Nervous Diseases, Queen Square, London WC1N 3BG.

Accepted 30 January 1978
}

thesis were right, this block would have had no effect. But guanethidine blocks are as successful in relieving the pain as are blocks of the sympathetic chain and ganglia. The previous hypothesis also neglected the state of extreme sensitivity of the region supplied by the damaged nerve in cases of causalgia, and the spreading of the state of sensitivity beyond the territory of the peripheral nerve involved, that often occurs.

In this paper we report the results of blocking the sympathetic supply to the affected region in a number of different painful conditions-in many of these, the block was performed merely because other kinds of therapy had failed. As guanethidine is effective in stopping the burning pain and hypersensitivity, and as it prevents the activity of noradrenaline at peripheral sites a new hypothesis is necessary; this will be considered in the Discussion.

\section{Methods}

Patients

There were 45 patients with 46 lesions in whom sympathetic blocks were carried out. Of these 45 patients, 13 had conditions in which blocking the sympathetic supply might have been expected to help; they had causalgia or reflex sympathetic dystrophy. The region of the body to which the sympathetic system was blocked were, faceseven, trunk-five, upper limb for 13 lesions in 12 patients, lower limb-21. In 29 patients only a block of the sympathetic chain and ganglia was done, in six patients only a block with guane- 
thidine was done (in one of these patients twice), and in 10 patients both kinds of blocks were done. The guanethidine block was carried out only in patients in whom the condition to be affected was below the knee or elbow.

\section{PROCEDURES}

Blocks of the chain or stellate ganglion were performed in the usual way with $2 \%$ lignocaine and $0.25 \%$ bupivacaine. The guanethidine blocks were done according to the technique described by Hannington-Kiff (1974). For the upper limb 12.5 to $15 \mathrm{mg}$ of guanethidine in $20 \mathrm{ml}$ of saline, and for the lower limb 15 to $20 \mathrm{mg}$ of guanethidine in $30 \mathrm{ml}$ of saline were injected intravenously. The cuff was kept on for 20 minutes before it was released.

During the blocks the temperature of the limb and of the opposite control limb was monitored by thermocouples. In blocks of the face and upper limb, evidence of block of the stellate ganglion was also looked for, with regard to the upper lid, the size of the pupil, conjunctival vessel dilatation, and blocking up of the affected side of the nose. If a block of the chain or ganglia produced any change in sensibility, indicating block of somatic nerve fibres, the block was repeated on another occasion, and the result of the first block was not included in the series.

\section{Results}

The clinical conditions for which sympathetic nerve blocks were performed, and the effect of the block in relieving pain are shown in Table 1 . Relief of pain means that pain was relieved for the duration of the block, and it often includes relief for hours or days afterwards. Sympathetic nerve block relieved the pain in reflex sympathetic dystrophy and Sudeck's atrophy, as would be expected. Sympathetic block was sometimes useful in painful amputations, post-herpetic neuralgia, and in the painful scar syndrome. In most other conditions it was unlikely to be useful but the number of cases is too small to have any significance. Apart from the group of reflex sympathetic dystrophy, the conditions which were helped by blocks were not those in which there was any obvious increase or decrease in sympathetic activity.

The relationship of the effectiveness of the block to the presence of hyperpathia is shown in Table 2. There were 31 patients with hyperpathia. In these 31 , the sympathetic block removed both the pain and the hyperpathia in 21 , it partially relieve $\mid$ both in two, and it failed to relieve the
Table 1 Effect of sympathetic block on painful conditions

\begin{tabular}{|c|c|c|c|}
\hline \multirow[b]{2}{*}{ Painful condition } & \multicolumn{3}{|c|}{$\begin{array}{l}\text { Effect of sympathetic block } \\
46 \text { lesions in } 45 \text { patients }\end{array}$} \\
\hline & $\begin{array}{l}\text { Pain } \\
\text { relieved }\end{array}$ & $\begin{array}{l}\text { Pain } \\
\text { unrelieved }\end{array}$ & $\begin{array}{l}\text { partially } \\
\text { relieved }\end{array}$ \\
\hline Reflex sympathetic dystrophy & 4 & 1 & 1 \\
\hline Sudeck's atrophy & 1 & 0 & \\
\hline Partial peripheral nerve lesion & 4 & 1 & \\
\hline $\begin{array}{l}\text { Compression of nerve or nerve } \\
\text { roots }\end{array}$ & 1 & 2 & \\
\hline Limb amputation & 3 & 4 & \\
\hline Post-herpetic neuralgia & 3 & 3 & \\
\hline Painful scar syndrome & 4 & 2 & \\
\hline $\begin{array}{l}\text { Tearing of brachial plexus } \\
\text { followed by amputation }\end{array}$ & 0 & 2 & \\
\hline $\begin{array}{l}\text { Carcinoma invading brachial } \\
\text { plexus }\end{array}$ & 1 & 0 & \\
\hline $\begin{array}{l}\text { Pain after Caldwell-Luc } \\
\text { operation }\end{array}$ & 0 & 1 & \\
\hline Pain after dental treatment & 0 & 1 & 1 \\
\hline Intramedullary spinal lesions & 0 & 2 & \\
\hline Arachnoiditis & 1 & 1 & \\
\hline $\begin{array}{l}\text { Dysaesthesiae after spinothalamic } \\
\text { lesion }\end{array}$ & 1 & 0 & \\
\hline Thalamic syndrome & 0 & 1 & \\
\hline Total & 23 & 21 & 2 \\
\hline
\end{tabular}

Table 2 Effect of sympathetic block on pain in hyperpathic and non-hyperpathic states

\begin{tabular}{llll}
\hline & $\begin{array}{l}\text { Patients } \\
\text { with } \\
\text { hyperpathia }\end{array}$ & $\begin{array}{l}\text { Patients } \\
\text { without } \\
\text { hyperpathia }\end{array}$ & Total \\
Effect on constant pain & 21 & 2 & 23 \\
Relieved & 2 & 0 & 2 \\
Partially relieved & 8 & 13 & 21 \\
Unrelieved & 31 & 15 & 46 \\
\hline Total & & & \\
\hline
\end{tabular}

hyperpathia and pain in eight. In 15 patients there was no hyperpathia; in this group of patients it relieved the pain in only two.

There is obviously a relation between hyperpathia and sympathetic activity. Hyperpathia and the pain associated with it will probably be relieved by sympathetic block. Pain will probably not be affected by sympathetic block unless hyperpathia accompanies the pain. When hyperpathia is present and the block does not relieve it, it also does not relieve the pain; it may occasionally relieve the hyperpathia without relieving the pain. This was illustrated in three patients in whom the relief of hyperpathia lasted for a few days, whereas the relief of pain lasted for one day. Most of the patients who had hyperpathia also had hyperaesthesia, and this was also removed by sympathetic block.

We, therefore, conclude that in cases such as causalgia it is not primarily burning pain for which sympathetic blocks or sympathectomy 
should be done but it is the state of hyperpathia with hyperaesthesia. It is true, nevertheless, that these patients nearly always describe some of the pains they have as burning pain.

What is meant by hyperpathia is that all stimuli cause a burning pain or a painful electric sensation. The sensation lasts longer than normally, and tends to irradiate out from the point stimulated. This occurs when the patient is stimulated, when he touches anything on purpose or by mistake, and when he moves; it prevents his moving the affected part. Many patients with this abnormal sensitivity of the skin also had deep, aching pain. The deeper tissues were also hyperpathic in their way. A sudden pressure, like a knock, or a deep sustained pressure increased the constant pain greatly, and it would go on hurting for many minutes. A few patients had the identical pain on stimulation that occurs when only nonmyelinated afferent fibres are conducting. Any adequate stimuli caused a burning, penetrating, lasting pain. These patients had a sphygmomanometer cuff put on the opposite limb so that they could experience the sensations when only nonmyelinated fibres were conducting, and could then make a comparison between the two pains. This kind of pain was also removed by sympathetic blocks.

Hyperaesthesia was defined by Noordenbos (1959) as follows: a state in which a stimulus which does not cause pain in normally innervated tissues, does cause pain in the affected region. Typically, a very slight stimulus such as a minimal distortion of the skin or the movement of a single hair causes pain. In these patients touching or tapping the area causes very painful sensations just like electric shocks. In some of the patients, the only outstanding abnormality is the pain with slight tactile stimuli. There may be no change in threshold to pain induced by radiant heat or pinprick. Deep pressure may cause pain, but, as is well-known, it is usually preferred to light touches.

When hyperpathia and hyperaesthesia are marked, every stimulus is dreaded, and all contacts are accompanied by a general alerting response, even though the patient expects the stimulus. As stimulation is continued, the pain increases so that finally the patient has the feeling that stimuli are still being applied after stimulation has ceased. This state is most obvious for mechanoreceptors, particularly for the most sensitive tactile receptors.

The tables record the effect of blocks on lesions and not on patients. The reason is that in one patient, there was more than one lesion, and the block affected one favourably and had no effect on the other. This difference between the effect on two separate lesions in one person should be noted, as some observers have thought that states such as reflex sympathetic dystrophy and causalgia occur only in certain types of people. It was also striking that in the patients with an amputated limb and pain in the stump, the sympathetic block relieved the pain in the region in which there were hyperaesthesia and hyperpathia, and not in the region where they were not present.

The effect on the pain and the state of abnormal sensitivity was immediate. When the hyperpathia and hyperaesthesia were removed by sympathetic block, the patient sometimes became aware of a diminution in sensibility, a numbness. Whether this occurred or not, there was an improvement in sensibility. The patients could feel the stimuli more clearly and were able to identify them more accurately. This improvement in sensibility occurs whenever and however the state of hyperpathia is removed (Nathan, 1960).

Usually when the block passed off, the pain, the hyperpathia, and the hyperaesthesia returned. But in 12 of the 45 patients, one or more blocks were therapeutically useful, and the pain was relieved for many days or even permanently.

Although there is a relationship between the $\mathbb{\Omega}_{\infty}$ presence of functioning sympathetic fibres ando hyperpathia with burning pain, it is obvious that the one does not depend upon the other. In three patients of this series and in many patients seen in the past, the pain and hyperpathia have returned within a year of a surgical sympathectomy; they have been seen to come on in a sympathectomised limb, the sympathectomy having been done for a vascular and not a painful condition. Thus the relationship between hyperpathia and the presence of active sympathetic nerves is not a necessary one.

The results given here are reported on account of their physiological importance. They are not to be held to be indications for lasting sympathectomy as therapy. But in patients with hyperaesthesia and hyperpathia, it may be worth trying the effect of blocking the sympathetic supply to the region, and if this relieves the state, then repeating it more than once. As is already generally recognised, blocks should be tried in causalgia, reflex sympathetic dystrophy, and Sudeck's atrophy. In five of the patients of this series, both the hyperpathic state and the pain were permanently relieved by sympathetic blocks.

When there is a complete lesion of a peripheral nerve or of a large amount of the brachial plexus, the hyperaesthesia and hyperpathia do not, of course, occur in the region of total denervation. If 
they are present, they are in the surrounding region in which innervation is incomplete. One also sees hyperpathia and more rarely hyperaesthesia on the skin flaps brought round to cover the stump after an amputation. When the hyperpathic state occurs with a partial lesion of a peripheral nerve, as is more common, there is some diminution in sensibility within the territory of this nerve, and there may be hyperpathia and hyperaesthesia both within and beyond this territory.

A striking feature of these states of sensitivity associated with lesions of peripheral nerves and with lesions in the central nervous system is the spread of hyperpathia. For instance, it might spread from the skin innervated by the ulnar nerve up the forearm and across the palm to encroach on the median area, and finally be within a whole forequarter of the body. This spread of the abnormal state was already recorded by Weir Mitchell over a hundred years ago. After a sympathetic block, the entire area of hyperpathia is removed. It is then easy for patients to notice an area of diminished sensibility and numbness, which is found to be restricted to the territory of the damaged nerve.

Guanethidine has membrane-stabilising effects and thus some local anaesthetic action. But in the way the substance was used here and with the amounts injected, the effects obtained were not due to local anaesthesia. Guanethidine never caused any change in sensibility, except for the removal of the hyperpathia, and no reduction in motor function. Moreover, the pain and hyperpathia were not stopped in all patients. In only one of the patients who had both kinds of blocks was there a difference in the effect of the block on pain and hyperaesthesia: in all other patients, either both stopped the pain and the hyperaesthesia or did not do so. But there were some differences in the effects of the two kinds of blocks. For example, cutaneous vasodilatation was immediate with blocks of the sympathetic chain, but it occurred after varying intervals with guanethidine-it might come on within minutes or within hours. The vasodilatation lasted only a few hours after blocking the chain, and it lasted for days after the guanethidine injection. The different effects of the two kinds of blocks is now being investigated.

The effect of blocking the sympathetic nerves in removing the hyperpathia and in improving sensibility is illustrated by a case. A 61 year old man (JE) had post-herpetic neuralgia in the maxillary division of the left cranial fifth nerve for three years. The pain was in a small area, within the region supplied by the maxillary division. It was maximal at the inner canthus of the eye. The pain was a constant burning, smarting, sore feeling. On examination, stroking with cottonwool within the maxillary division of the nerve was not felt, and he could not distinguish the two ends of a pin; he could not feel the pin at all until it had indented the skin considerably. A wooden rod of $3 \mathrm{~g}$ dropped onto the cheek was not felt. There was more sensibility with hyperpathia over the left ala nasi and on the upper lip on the left, but not on the lip margin nor on the gums. In these regions stroking with cottonwool was felt, and all tactile stimuli "felt more" than on the right side of the face. In the affected region, all stimuli were painful and burning, and caused flinching but pinpricks felt less sharp than in normal areas.

A block of the stellate ganglion was carried out on three occasions, each with the same result. The hyperpathia was removed, the two sides of the face subjectively felt the same and normal, and the patient ceased to be continually aware of the left side of the face. On examination, sensibility was normal. No touches or strokes with cottonwool were missed, the two ends of a pin were distinguished, and the prick felt like a normal prick. There was no difference in sensibility between the areas over the ala nasi and upper lip and the rest of the face. The patient considered that this was a far better block than a previous block of the infraorbital nerve as the sympathetic block restored sensibility to normal, and the somatic nerve block had left him with a feeling of numbness and of swelling of the face.

After the third occasion on which this block was carried out, the patient was free from pain and hyperaesthesia for 14 days. After two more stellate blocks the pain in the eye went away, and the rest of the pain gradually subsided.

The failure of sympathetic block in removing burning pain is illustrated by the next case. $\mathrm{CN}$, aged 47 years, fell off a ladder in her kitchen at Christmas 1974 and fractured the left maxilla. When we saw the patient in January 1976, radiography showed a depression of the left orbital margin consistent with an old zygoma and antral fracture. The pain had started immediately after the injury and was getting worse. It was in the upper lip, in the gum of the left upper jaw, and in the left malar region, reaching up to the nose. It consisted of "a terrible burn, like being scalded," and also some aching below and behind the left eye. She was unaware of any numbness of her face. In the distribution of the left infraorbital nerve, including its palpebral, nasal, and labial 
divisions, and the middle and anterior superior dental nerves, there was a state in which all contacts made her flinch; they caused tingling and painful stinging. Deep pressure in the affected region was painful. Cottonwool was felt equally on the two sides of the face, but pinprick felt more penetrating and painful in the region of the nerve lesion. We concluded that there was a partial lesion of the infraorbital nerve, which was being compressed in the bony canal.

A sympathetic block had no effect on her pain nor on sensibility.

\section{Discussion}

This discussion will be concerned mainly with the cases in which blocking the sympathetic system removes pain, hyperaesthesia, and hyperpathia. The conclusions from this study are as follows:

1. Sympathetic blocks do not achieve their effects by acting at the site of the lesion of a peripheral nerve. This is shown by the removal of pain and abnormal sensitivity by blocks of the sympathetic nervous system in cases in which the pain and sensitivity are caused by a lesion in the spinal cord, in the posterior root ganglia, around the nerve roots, or on a peripheral nerve proximal to the sphygmomanometer cuff in a guanethidine block. This shows that the former hypothesis of the pain of causalgia being caused by ephaptic transmission at the site of the lesion on the nerve is wrong. Blocking the emission of noradrenaline along the course of peripheral nerve fibres is unlikely to be the cause of the relief of the hyperpathia, hyperaesthesia, and pain. The reason for stating this is that the effects of sympathetic block are the same in the face and in the limbs. Yet in the face the sympathetic fibres accompany the arteries and not the peripheral nerves, as they do in the limbs. It is concluded that the site at which effects are obtained with sympathetic blocks is the noradrenergic effector target in the skin and deeper tissues.

2. There is a good, but not necessary, correlation between blocking sympathetic fibres and the removal of pain, hyperpathia, and hyperaesthesia.

The most common kind of pain accompanying hyperpathia is burning pain, and so the commonest pain relieved by sympathetic blocks is burning pain. Other pains associated with hyperpathia, such as shoots of pain, and pain described as the digging in of knives, are usually relieved when hyperpathia is relieved. A deep pain of a constant nagging aching kind is least likely to be relieved.

3. In some of the patients in whom sympathetic blocks relieve the pain and abnormal sensitivity, there is no evidence of abnormal sympathetic function. There is normal sweating and vascular state and normal skin conductance.

4. In the painful states associated with hyperaesthesia and hyperpathia, the lesion in the periphery has induced abnormal functioning in the central nervous system, presumably at spinal level. This statement is based on the fact that tactile stimulation causes pain and on the fact of the spread of pain and hypersensitivity beyond the original territory of the lesion.

It seems unlikely that the output of noradrenaline could change the characteristics of peripheral endings of types I and II mechanoreceptors and hair receptors so that activation of these receptors causes pain, or that it could lower the threshold of delta or nonmyelinated nociceptors so that they would be excited by minimal mechanical stimuli. It is more likely that constant firing has caused a change in central activity. This might be a relaxation of tonic inhibition or increased facilitation. Either or both of these or other mechanisms could affect neurones of lamina 5 on which mechanoreceptor and nociceptor fibres converge; in this case these neurones might be fired by slight tactile stimulation.

In these states, there is commonly an eventual $\stackrel{\mathbb{Q}}{\Phi}$ spread of hyperpathia and hyperaesthesia beyondo the original limits of the affected region; it can even occupy a whole quarter of the body. Thiso secondary region of hyperpathia and hyperaesthesia is also removed by blocking the sympathetic supply of the peripheral region. This spreading of pain and abnormal sensitivity indicates a spreading of the abnormal central state from the original region of input into the neighbouring caudal and cranial regions. This might be in the substantia gelatinosa or in lamina 5 .

5. In this way, one supposes that blocking the action of noradrenaline in the peripheral tissues reverses an abnormal state in the central nervous system, presumably at segmental level.

Three questions require an answer:

Why does the presence of the normal transmitter, noradrenaline, eventually induce a state of disinhibition or facilitation in the spinal cord?

Why, in the chronic painful states under discussion, does the central disinhibition or facilitation eventually spread from its original site to the neighbouring region of the posterior horns?

Why are some lesions and not others affected in this way?

Facts known about the sympathetic system that might help in answering these questions will now be considered. 
There are two important papers, rarely quoted because they are in Russian, by Motsnyi and Bagramova (1972) and Bagramova et al. (1976). They show that stimulation of the sympathetic nervous system and the emission of adrenaline influence the dorsal root potential and the dorsal root reflex, in the cat. The effects are complicated. Motsnyi and Bagramova (1972) at first observed depolarisation of primary afferent nerve terminals while stimulating the sympathetic chain. They concluded "that the mediator liberated during stimulation of the sympathetic trunk potentiates depolarisation of afferent fibres." As often appears with observations and experiments on the sympathetic system, the effects obtained from stimulating the sympathetic chain are not always the same. This was seen in the later paper by Bagramova et al. (1976). In $65 \%$ of the experiments, preceding excitation of the sympathetic chain caused presynaptic depolarisation, in $25 \%$ it caused hyperpolarisation followed by depolarisation, and in $10 \%$ it caused hyperpolarisation. Depolarisation was accompanied by an increased dorsal root reflex in cutaneous and in mixed nerves, which they called the antidromic response. Thus they concluded that one of the factors affecting the membrane potential of primary afferent fibres was sympathetic activity and circulating adrenaline.

Although the discovery of the effects of sympathetic activity on the input by primary afferent fibres into the spinal cord is interesting, it is not applicable to our cases, and it does not contribute towards answering the questions that arise from our material. The guanethidine blocks show that the effects we obtained were the result of blocking sympathetic nerves at their endings.

One piece of work suggests that sympathetic activity could affect conduction in the somatic nerves. Bülbring and Whitteridge (1941) found that the intra-arterial injection of adrenaline increased the size of the action potential of the sciatic nerve in the cat. The $\alpha-\beta$ spike was increased up to $100 \%$. This observation does not help to answer the questions posed above, nor is it relevant to our results, for, as has been mentioned, we got the same results in the face as in the limbs; and the sympathetic nerves do not accompany the somatic nerves in the face. Further, it seems unlikely that a function of sympathetic nerve fibres is to amplify conduction in peripheral somatic nerves; for if it were, all somatic nerve fibres would be accompanied by sympathetic fibres.

As the relief of pain was associated with relief of hypersensitivity, one is ready to favour any suggestion that a function of the sympathetic system is to control the sensitivity of peripheral receptors. The evidence in support of this belief will now be reviewed. In our opinion, it is inadequate. Moreover, obvious states of sympathetic overactivity and underactivity are not accompanied by subjective nor objective changes in sensibility.

General interest greeted the paper by Loewenstein (1956) on the effects of sympathetic stimulation and the application of noradrenaline and adrenaline to the cutaneous receptors of the frog, and these experiments became even more interesting when Loewenstein and AltamiranoOrrego (1956) repeated them on the cat. Loewenstein (1956) showed that "stimulation of the sympathetic nerve supply to an isolated frog's skin or the application of adrenaline or noradrenaline" lowered the threshold of a mechanoreceptor so much that it eventually fired spontaneously, and Loewenstein and Altamirano-Orrego (1956) showed that it did the same to a Pacinian corpuscle in the cat's mesentery. Chernetski (1964) greatly amplified this work on the frog, and he too found that sympathetic stimulation increased the discharge from mechanoreceptors and also from gustatory receptors. Eldred et al. (1960) found in the cat that tendon organs and muscle spindles reacted to sympathetic stimulation and to the intra-arterial injection of noradrenaline and adrenaline. There was a slight increase in firing followed by cessation of firing. The cat's vibrissae, according to Nilsson (1972), fire less frequently with mechanical stimuli during sympathetic stimulation, although the effect was slight. Edwall and Scott (1971) showed in the cat that stimulation of the sympathetic supply of the dentine and pulp of the tooth caused at first an increase and then a decrease in the firing of the somatic afferent nerve fibres. Matthews (1976) carried out the same experiments, and also injected noradrenaline or adrenaline by close arterial iniection. The application of a solution of sodium chloride, that is painful in man, to the dentine of the cat caused a low frequency discharge in the afferent nerve fibres. The addition of sympathetic stimulation caused "a marked increase of up to 30 -fold in the discharge evoked:" the same effect resulted from the intra-arterial injection of adrenaline and noradrenaline. The effect was stopped by alphablockers and not by beta-blockers.

If the sympathetic system were normally controlling the sensitivity of receptors, evidence of this would have been obvious throughout neurology. Patients with increased sympathetic activity as in hyperhidrosis and in hyperthyroidism would show 
one sort of sensory abnormality, and patients with decreased activity as in hypothyroidism would show the opposite sort. In the face of this lack of evidence of the sympathetic system affecting sensory receptors, the few recherche examples reported in physiological literature are inadequate to maintain this general hypothesis.

More relevant to our material are the experiments carried out by Wall and Gutnick (1974) to throw light on the pain associated with amputations. They induced a neuroma on the sciatic nerve of the rat. Eventually the smallest fibres of this neuroma were constantly discharging impulses. These small fibres were fired by mechanical excitation of the neuroma. More important for our cases, their firing was induced and increased by noradrenaline, including noradrenaline passing in the blood supply; it was stopped by the alphablocker phentolamine and unaffected by isoprenaline and other beta-agonists. The activity was stopped quickly by cutting off the blood supply. The conditions of a neuroma at the end of a cut nerve are probably the same as those of a partial lesion on the course of a peripheral nerve in which fibres have been cut or damaged. It is likely that there are similar noradrenergicsensitive nerve fibres in the kinds of cases being discussed here, in which the pain and sensitivity are removed by sympathetic blocks. In this case, there must be constant firing of small nerve fibres with increased firing whenever the damaged nerve is touched or moved. Against this assumption are the many cases of causalgia in which the pain comes on immediately or within hours of the injury; in such cases, there could hardly be any outgrowth of fibres. Further, we do have to explain why outgrowing of small fibres sensitive to noradrenaline is present in some lesions and absent in others.

It seems that the condition of hypersensitivity depends on an input in the largest cutaneous fibres, the beta group; and, as in the cases under discussion, the burning pain is associated with this cutaneous hypersensitivity, it is likely that this pain also depends on the input in these fibres. Anything that inactivates these fibres temporarily removes hyperaesthesia, hyperpathia, and the associated burning pain.

In this discussion of the pain induced or maintained by sympathetic nerve fibres, and its relief by the removal of sympathetic activity, we have not yet mentioned painful states brought on by sympathectomy. There is a state, sometimes called sympathalgia, of continual pain coming on 10-18 days after surgical sympathectomy, in which all deep tissues are tender on moderate pressure.
The patient says when he is examined that all stimuli feel different, though he cannot explain in what way this is so. The limb gets tired more easily than the opposite limb; it feels heavier, weaker, and it is an effort to move it. When the sympathectomy includes the stellate ganglion, the eye is mainly affected, and it is painful and tender on pressure. This state usually clears up in about six weeks, but it may be more severe and last much longer.

Although surgeons who do sympathectomies must see many of these cases, there is little in the literature about the syndrome. It has been reported by Pette $(1927,1928)$ and by Tracy and Cockett (1957). What is important to note is that Tracy and Cockett showed sympathetic overactivity in the regions in which there were remaining sympathetic fibres. There was pain and tenderness in the region where there was a partial sympathectomy and in these regions there was excessive sympathetic activity. The tenderness is over neurovascular bundles and large arteries, and the muscles are tender. This state, then, seems to be due to sympathetic denervation sensitivity in a region in which there is partial sympathetic denervation. The clinical picture is totally unlike the state of hyperpathia and hyperaesthesia, usually accompanied by normal sympathetic activity, that is being described here.

One of the main features of the states cured by blocking the sympathetic outflow is the spread of the hypersensitivity beyond the original boundaries of the region innervated by the damaged nerve. It is apparent that there is a spreading state of disinhibition or facilitation in the posterior horn originating at the site of input from the damaged peripheral nerve fibres. It appears to be, as has been argued above, induced and kept going by the input in the largest afferent fibres. It depends on this input, for it is readily removed when this input is changed or removed. It appears to be the tactile fibres and mechanoreceptors that in these states are causing pain and the reactions associated with pain.

This discussion is full of unanswered questions. Finally, the few facts should be restated. Blocking the sympathetic fibres, which prevents noradrenaline release, stops the hyperaesthesia, hyperpathia, and pain in certain painful states; yet these states do not often show evidence of sympathetic overactivity. The conditions relieved by sympathetic blocks include cases in which the lesion is within the central nervous system. Thus, stopping the emission of the normal sympathetic transmitter stops the firing of peripheral nerve fibres and that stops the abnormal function within 
the central nervous system. The abnormal state is kept going by mechanoreceptors and the large afferent fibres. The sympathetic outflow is acting on these fibres or receptors to cause the input to the spinal cord which causes a state of disinhibition or facilitation spreading from the original site of input.

We would like to thank our colleagues at the National Hospital for Nervous Diseases for referring patients to us, Dr P. G. Wilson for stimulating discussions of the subject, and Dr Marion C. Smith for helpful criticisms in writing this paper.

\section{References}

Bagramova, M. A., Tisova, R. A., and Mantulo, P. M. (1976). Changes in the posterior root potential and excitability of primary afferent terminals on the cat spinal cord evoked by repetitive stimulation of the sympathetic trunk. Fiziologicheskii Zhurnal I. $M$. Sechenova, 62, 137-140. In Russian.

Bülbring, E., and Whitteridge, D. (1941). The effect of adrenaline on nerve action potentials. Journal of Physiology, 99, 201-207.

Chernetski, K. E. (1964). Sympathetic enhancement of peripheral sensory input in the frog. Journal of Neurophysiology, 27, 493-515.

Doupe, J., Cullen, C. R., and Chance, G. Q. (1944). Post-traumatic pain and the causalgic syndromes. Journal of Neurology, Neurosurgery, and Psychiatry, 7, 33-48.

Edwall, L., and Scott, D. (1971). Influence of changes in microcirculation on the excitability of the sensory unit in the tooth of the cat. Acta Physiologica Scandinavica, 82, 555-566.

Eldred, E., Schnitzlein, H. N., and Buchwald, J. (1960). Response of muscle spindles to stimulation of the sympathetic trunk. Experimental Neurology, 3, 13-25.
Hannington-Kiff, J. (1974). Intravenous regional sympathetic block with guanethidine. Lancet, 1, 1019-1020.

Loewenstein, W. R. (1956). Modulation of mechanoreceptors by sympathetic stimulation. Journal of Physiology, 136, 40-60.

Loewenstein, W. R., and Altamirano-Orrego, R. (1956). Enhancement of activity in a Pacinian corpuscle by sympathomimetic agents. Nature, 178, 1292-1293.

Matthews, B. (1976). Effects of stimulation on the response of intradental nerves to chemical stimulation of dentin. In Advances in Pain Research and Therapy, Vol. 1. Edited by J. J. Bonica and D. Albe-Fessard. Raven Press: New York.

Motsnyi, P. E., and Bagramova, M. A. (1972). Sympathetic influences on presynaptic inhibition of the dorsal spinal cord potential. Fiziologicheskii Zhurnal I. M. Sechenova, 58, 697-701. In Russian.

Nathan, P. W. (1947). On the pathogenesis of causalgia in peripheral nerve injuries. Brain, 70, 145171.

Nathan, P. W. (1960). Improvement in cutaneous sensibility associated with relief of pain. Journal of Neurology, Neurosurgery, and Psychiatry, 23, 202206.

Nilsson, B. Y. (1972). Effects of sympathetic stimulation on mechanoreceptors of cat vibrissae. Acta Physiologica Scandinavica, 85, 390-397.

Noordenbos, W. (1959). Pain. Elsevier: Amsterdam.

Pette, H. (1927). Das Problem der wechselseitigen Beziehungen zwischen Sympathikus und Sensibilität. Deutsche Zeitschrift für Nervenheilkunde, 100, 143-164.

Pette, H. (1928). In Discussion on O. Schwab's Die Topik der Sensibilitäts Störungen bei Läsionen der sensiblen Leitungsbahnen und Zentren. Deutsche Zeitschrift für Nervenheilkunde, 101, 255-256.

Tracy, G. D., and Cockett, F .B. (1957). Pain in lower limbs after sympathectomy. Lancet, 1, 12-14.

Wall, P. D., and Gutnick, M. (1974). Ongoing activity in peripheral nerves: the physiology and pharmacology of impulses originating from a neuroma. Experimental Neurology, 43, 580-593. 